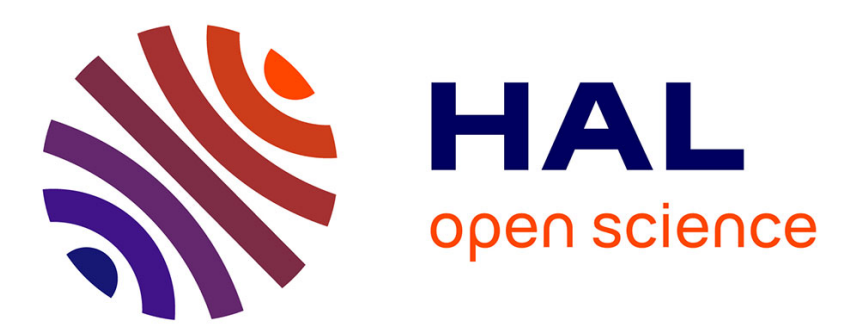

\title{
Numerical modelisation of the scattering of an acoustic wave from a wedge immersed in a fluid
}

S. Chadli, S. Melliani, J. Colombeau, B. Perrot, M. de Billy

\section{To cite this version:}

S. Chadli, S. Melliani, J. Colombeau, B. Perrot, M. de Billy. Numerical modelisation of the scattering of an acoustic wave from a wedge immersed in a fluid. Journal de Physique IV Proceedings, 1994, 04 (C5), pp.C5-701-C5-704. 10.1051/jp4:19945150 . jpa-00252829

\section{HAL Id: jpa-00252829 https://hal.science/jpa-00252829}

Submitted on 1 Jan 1994

HAL is a multi-disciplinary open access archive for the deposit and dissemination of scientific research documents, whether they are published or not. The documents may come from teaching and research institutions in France or abroad, or from public or private research centers.
L'archive ouverte pluridisciplinaire HAL, est destinée au dépôt et à la diffusion de documents scientifiques de niveau recherche, publiés ou non, émanant des établissements d'enseignement et de recherche français ou étrangers, des laboratoires publics ou privés. 


\title{
Numerical modelisation of the scattering of an acoustic wave from a wedge immersed in a fluid
}

\author{
S.L. CHADLI, S. MELLIANI, J.F. COLOMBEAU, B. PERROT* and M. DE BILLY** \\ ENS de Lyon, 46 Allée d'Italie, 69364 Lyon cedex 07, France \\ * Université de Bordeaux I, 33405 Talence, France \\ ** GPS, Universités Paris 6 et Paris 7, 2 place Jussieu, 75251 Paris cedex 05, France
}

\begin{abstract}
In the present paper, we propose a numerical technique describing the scattering of an acoustic wave interacting with a sharp wedge immersed in water considered as homogeneous. The calculations are limited to the angular variations of the acoustic field scattered in a plane perpendicular to the edge of the wedge. The theoretical angular plots which point out maxima in particular directions are compared with the experimental measurements. We notice a good agreement between the two determinations.

\section{Résumé}

Nous décrivons dans ce papier une méthode de calculs numériques permettant de décrire la diffusion d'une onde acoustique plane interagissant avec un coin immergé dans un fluide considéré comme homogène. Les calculs théoriques, qui sont limités aux variations angulaires du champ acoustique diffusé dans un plan perpendiculaire à l'arête du coin, révèlent l'existence de maxima de pression dans des directions particulières. Les valeurs theoriques des angles correspondant à ces maxima sont comparées avec celles obtenues expérimentalement. On note un bon accord entre ces deux déterminations.
\end{abstract}

\section{Introduction}

Durant ces dernières années, un nombre relativement limité de travaux ont été consacrés à l'étude de l'interaction d'une onde acoustique par un dièdre plein, élastique, formé de deux demiplans semi-infinis $[1,3]$. Nous nous proposons de décrire une méthode numérique permettant de mettre en évidence l'influence de l'angle du dièdre sur les diagrammes de diffusion observés dans un plan perpendiculaire à l'arête d'un dièdre immergé et selon deux configurations géométriques différentes:une configuration dite "normale" dans laquelle la direction moyenne du faisceau incident est perpendiculaire à l'un des demi-plans formant le dièdre et une configuration dite "tangentielle" pour laquelle la direction moyenne du faisceau incident est contenue dans l'un des demi-plans définissant l'échantillon (Fig. 1). Il existe en général un bon accord entre les résultats théoriques et les diagrammes angulaires relevés expérimentalement.

\section{Modèle théorique}

En utilisant la méthode développée pour un dioptre acoustique fluide / fluide [4] et en l'adaptant au cas du dioptre fluide / solide élastique, il a été réalisé un schéma numérique permettant de calculer les variations angulaires du maximum absolu de pression acoustique diffusée par un dièdre élastique immergé, à une certaine distance de l'arête du dièdre. Dans ce schéma nous nous 
plaçons dans le cas de l'acoustique linéaire et prenons les équations en variables de Lagrange [5] qui s'ecrivent respectivement :

-- pour le liquide

$$
\left\{\begin{array}{l}
\partial_{t} p(x, t)+c_{l i q}^{2} \cdot \rho_{l i q} d i v_{x} U(x, t)=0 \\
\partial_{t} U(x, t)+\frac{1}{\rho_{l i q}} \cdot \operatorname{grad}_{x} p(x, t)=0
\end{array}\right.
$$
où p est la pression acoustique, $U$ le vecteur vitesse, $\rho_{i j}$ la densité du liquide et $c_{\text {trig }}$ la vitesse de
propagation de l'onde dans le liquide;

-- pour le solide élastique

$$
\left\{\begin{array}{l}
\partial_{t} \Sigma(x, t)-\lambda d i v_{x} U(x, t) \cdot 1 \mathrm{I}-\mu \cdot\left(\operatorname{grad}_{\mathrm{x}} U+\left(\operatorname{grad}_{\mathrm{x}} U\right)^{\mathrm{T}}\right)=0 \\
\partial_{t} U(x, t)-\frac{1}{\rho_{s o l}} \cdot d i v_{x} \Sigma(x, t)=0
\end{array}\right.
$$

où $\Sigma$ est le tenseur des contraintes, $U$ le vecteur vitesse, $\rho_{\text {stl }}$ la densité du solide, $\lambda$ et $\mu$ ses coefficients de Lamé et la matrice unité. Le cas du milieu liquide apparait comme un cas particulier du solide avec $\mu=0$, ce qui permet de considérer tout l'espace comme un espace unifié, dont les coefficients sont continus à l'interface liquide-solide. On peut montrer alors que la résolution du problème se réduit à un système à cinq équations scalaires, à coefficients constants dans chaque milieu. Pour l'obtention des résultats présentés ici, nous avons utilisé une méthode de directions alternées qui consiste à traiter le système comme la juxtaposition successive de deux systèmes unidimensionnels selon les deux dimensions d'espace. Chaque système unidimensionnel est discrétisé par un schéma aux différences finies classique (obtenu par développement de Taylor à l'ordre deux). Dans le problème qui nous intéresse, le point le plus délicat est celui du traitement de l'interface, car il peut influencer le résultat de façon significative [6]. On se contente de définir sur l'interface les valeurs des coefficients du système comme des moyennes des coefficients de chacun des milieux (eau,duraluminium), après avoir vérifié que, pour les valeurs des coefficients utilisés, ce choix donne des résultats en accord avec l'étude en référence [6]. Le schéma utilisé sera détaillé prochainement dans la réf. 7. On obtient finalement une méthode numérique simple et efficace. La condition initiale retenue est de considérer une impulsion incidente vers l'arête de forme gaussienne en pression et ayant une valeur significativement non nulle sur un disque d'environ $10 \mathrm{~h}$ de diamètre (le plan est découpé en mailles de côté h arbitrairement choisi). Les courbes isobares du maximum de la pression acoustique sur l'intervalle de temps d'étude sont visualisées dans une fenêtre carrée de coté $80 \mathrm{~h}$ (Fig. 1). Cette fenêtre se situe très près de l'arête. On postule que les directions des faisceaux "diffusés", sont données par les directions des droites orthogonales aux courbes isobares. Ces directions peuvent être facilement définies grâce à un processus itératif. Cette détermination se fait en général le plus loin possible de l'arête pour se trouver le plus possible dans les conditions expérimentales.
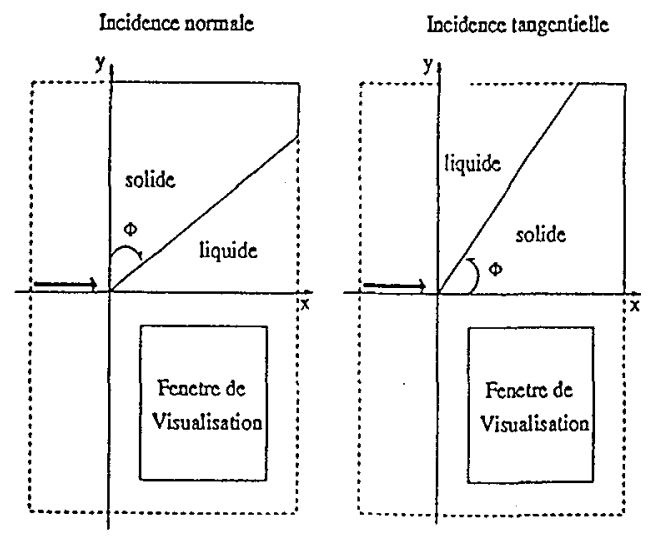

Fig. 1 Définition de la géométrie de l'expérience. 


\section{Comparaison théorie-expérience}

Une comparaison entre les résultats numériques et les mesures expérimentales correspondantes [2] est proposée sur les figures 2 et 3 pour trois valeurs de l'angle du dièdre. Il ressort de cette comparaison que quel que soit le type de configuration, il existe un bon accord entre le nombre et la position des directions angulaires indiquant une pression maximale diffusere (Cf. Tableaux 1 et 11). Il semble en revanche que l'amplitude relative des deux faisceaux réémis ne soit pas respectée dans le cas de l'incidence tangentielle (Cf. Fig. 2). Ce désaccord pourrait s'expliquer par les conditions initiales qui ne sont pas les mêmes entre la théorie et l'expérience.
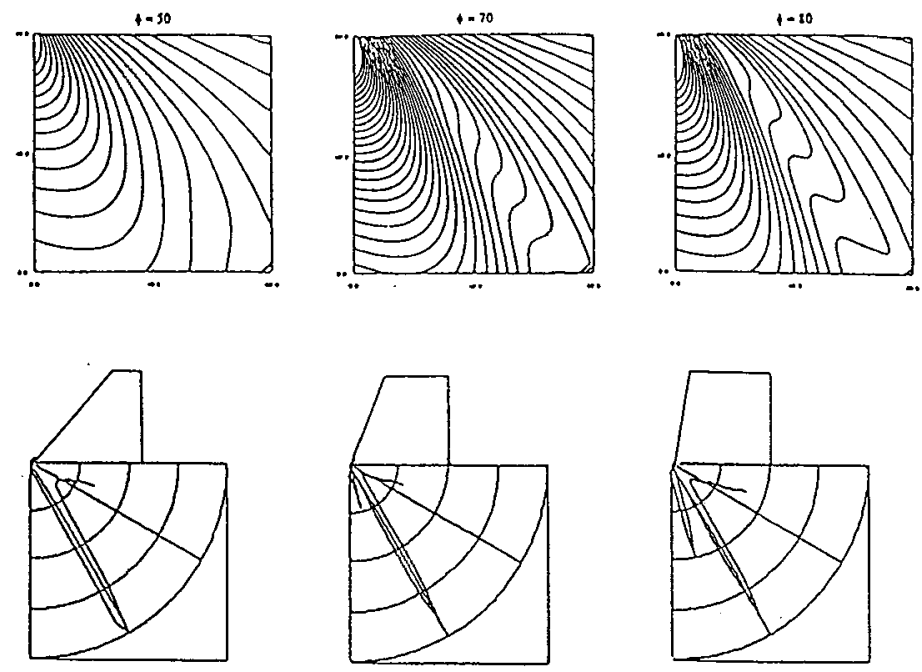

Fig.2 Comparaison théorie-expérience. Cas de l'incidence tangentielle.
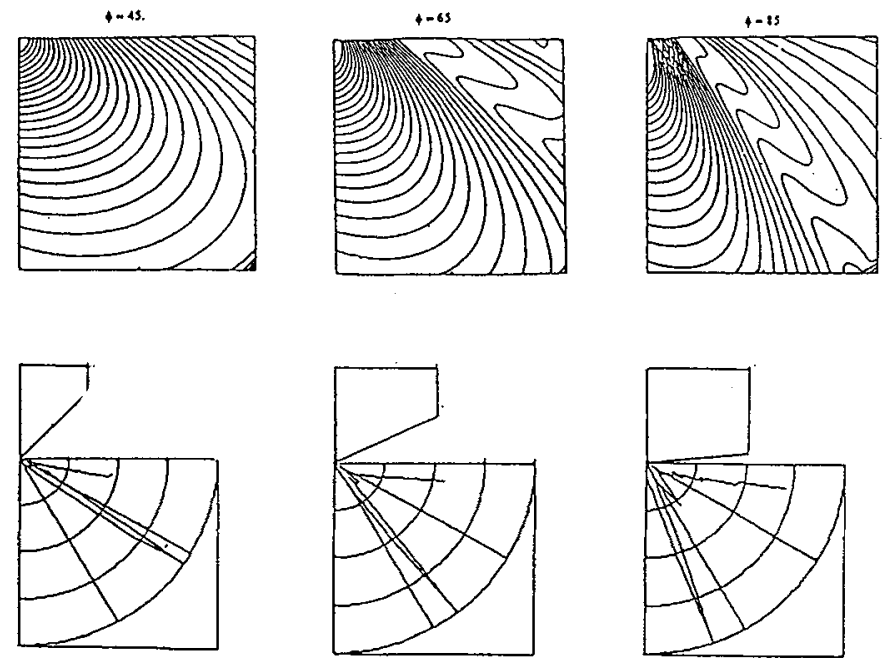

Fig. 3 Comparaison théorie-expérience. Cas de l'incidence normale. 


\section{Conclusion}

Ces résultats confirment la validité du modèle théorique qui doit pouvoir se généraliser à d'autres types de géométries et de cibles présentant ou non des discontinuités.

\section{Références}

1. C. Locqueteau Thèse de l'Université de Marseille II, 1992.

2. J.F. Piet et M. de Billy, J.A.P. 69, 6904-6909 (1991).

3. J.F. Piet et M. de Billy, Jl. de Physique Vol. 2 C1-683, Arcachon (1992).

4. Y.A. Barka, J.F. Colombeau et B. Perrot, Jl.d'Acou. 2,333-346 (1989).

5. B. Poirée, La Diffusion Acoustique, N. Gespa - Cedocar (Chap. 1).

6. J.F. Colombeau, Lecture Notes in Math. 1532, Springer Verlag (1992) Berlin-HeidelbergNew York p. 28.

7. S. Chadli, Thèse de Doctorat (à soutenir en 1994).

$\begin{array}{ccc}\text { gle cill dièdre } & \begin{array}{c}\text { angle de } \\ \text { diffusion\# }\end{array} & \begin{array}{l}\text { angle de } \\ \text { diffusion+ }\end{array} \\ 90^{\circ} & 15.0^{\circ} & 16.0^{\circ} \\ 80^{\circ} & 16.0^{\circ} & 15.0^{\circ} \\ 70^{\circ} & 15.0^{\circ} & 15.0^{\circ} \\ 60^{\circ} & 14.0^{\circ} & 13.0^{\circ} \\ 50^{\circ} & 12.5^{\circ} & 12.0^{\circ} \\ 40^{\circ} & 10.0^{\circ} & 11.0^{\circ} \\ 30^{\circ} & 6.0^{\circ} & 8.0^{\circ}\end{array}$

\# détermination à partir des données expérimentales

+ détermination à partir du modèle numérique

Table I Différentes valeurs des angles de diffusion correspondant à un maximum de pression en fonction de l'angle du dièdre dans la configuration géométrique : "incidence normale".

angle du dièdre

( $\theta)$

$80^{\circ}$

$70^{\circ}$

$50^{\circ}$

$$
\text { angle de }
$$

diffusion\#

$$
13.0^{\circ}
$$

$31.0^{\circ}$

$30.0^{\circ}$

$29.0^{\circ}$ angle de

diffusion+

$15.0^{\circ}$

$32.0^{\circ}$

$31.0^{\circ}$

$28.0^{\circ}$

\# détermination à partir des données expérimentales

+ détermination à partir du modèle numérique

Table II Différentes valeurs des angles de diffusion correspondant à un maximum de pression en fonction de l'angle du dièdre dans la configuration géométrique : "incidence tangentielle". 